\title{
Pesquisa Qualitativa em Marketing e suas Variações: Trilhas para Pesquisas Futuras ${ }^{(1)}$
}

\author{
Valter Afonso Vieira \\ Fernando Tibola
}

\section{Resumo}

A pesquisa qualitativa vem sendo crescentemente utilizada em marketing para desvendar os pensamentos e as motivações mais subjetivas do consumidor. Neste trabalho, após realizarmos uma breve revisão teórica do que é a pesquisa qualitativa em marketing, nosso objetivo concentrase em proporcionar e aventar as principais e mais empregadas variações dessa metodologia de pesquisa no campo do marketing, a saber: Entrevista de Profundidade, Grupos de Foco, Técnicas Projetivas, Pesquisa Observacional, ZMET (Zaltman Metaphor Elicitation Technique), Autodriving e Filmes. No tópico seguinte propomos uma tabela comparativa englobando as vantagens e desvantagens de cada variação da pesquisa qualitativa aplicada em marketing. Após as considerações finais delimitamos novas possibilidades para investigações futuras que acreditamos, possam ajudar ainda mais na utilização dessa técnica por profissionais de pesquisa no contexto nacional.

Palavras-chave: pesquisa de marketing; métodos qualitativos; análise de técnicas.

\section{Abstract}

The qualitative research is been increasingly used in marketing to unmask the thoughts and motivations more subjective from the consumer. After we accomplish an abbreviation theoreticalrevision of what is the qualitative research in marketing, our main goal concentrate on to providing and to fanning the main and more used variations of that research methodology in marketing - In depth interview, focus group, projective techniques, observational research, ZMET (Zaltman Metaphor Elicitation Technique), autodriving and films. In the following topic we propose a comparative table including the advantages and disadvantages of each qualitative research variation applied in marketing. After the final considerations, we suggest possible applications for future investigations that can help even more the use of that technique for research professionals in the national context.

Key words: marketing research; qualitative methods; technique analysis. 


\section{INTRODUÇÃO}

Visando descrever e analisar o homem e suas organizações culturais, a antropologia cultural defrontou-se com um objeto que resistia aos métodos estatísticos tradicionais empregados pela pesquisa quantitativa (BACELLAR, 2000). Com a existência desta dificuldade de analisar o homem mais profundamente surgiu a Pesquisa Qualitativa, que propõe desvendar um objeto subjetivo do pensamento humano.

Segundo o artigo de Bacellar (2000), a pesquisa qualitativa foi sendo gradativamente adotada também pela Sociologia, Psicologia, Educação e outras áreas dos estudos sociais, fazendo eclodir diversas técnicas para o método qualitativo.

Especificamente nas ciências sociais, por exemplo, o marketing se tornou um importante usuário da pesquisa qualitativa para subsidiar as ações de marketing. Em 1997, na Europa foram gastos 10.4 bilhões de euros em pesquisas de mercado, sendo que desse total $9 \%$ foi destinado exclusivamente para a pesquisa qualitativa (ZIMMERMAN; SZENBER, 2000).

Assim, visando contribuir para a discussão e enriquecimento dessa metodologia no contexto nacional, este artigo tem por objetivo apresentar e arrazoar especificamente as principais variações de pesquisa qualitativa (ou técnicas qualitativas) mais utilizadas em marketing.

Nós justificamos o presente trabalho com algumas características excepcionais que o discutem; entre elas destacamos:

. Primeiro, o fato de ser um trabalho de análise e comparação de múltiplas técnicas qualitativas juntas; em nível nacional, não encontramos nada nesse sentido ${ }^{(2)}$.

- Segundo, a importância dos trabalhos de comparação entre técnicas qualitativas já foi insinuado como necessário e importante para a pesquisa de marketing (CATTERALL, 1998).

- Terceiro, a aplicação gerencial e usufruto prático que profissionais e agências de pesquisa poderão fazer com os achados e colaborações, aqui discutidos.

- Quarto, a contribuição principal de explicar as vantagens e desvantagens de cada técnica, por meio de um quadro ilustrativo. 
- Quinto, o fato de que algumas das variações de pesquisa qualitativa, aqui debatidas, estão em caráter inicial de teste e uso em nível nacional e outras ainda nem começaram os testes, incentivando assim suas aplicações.

- Por fim, o fato de que o trabalho traça novas sugestões para futuras pesquisas em técnicas qualitativas.

Este artigo está dividido em cinco partes, incluindo essa breve introdução. $\mathrm{Na}$ segunda parte apresentamos uma revisão teórica do que é a pesquisa qualitativa em marketing, algumas de suas definições e conceitos, e os objetivos que a norteiam. Na terceira parte são discutidas, isoladamente, as principais variações e características dessa metodologia de investigação. No tópico posterior, propomos, com base na revisão de literatura, uma tabela comparativa, englobando as vantagens e desvantagens de cada variação da pesquisa qualitativa empregada em marketing. As considerações finais, bem como sugestões e direções para pesquisas futuras contemplam a parte final deste trabalho.

\section{A Pesquisa Qualitativa: Suas Definições e Objetivos}

Uma das tarefas críticas dos administradores de marketing é a tomada de decisão (KOTLER, 2000). Tomar decisões envolve não apenas a solução de problemas, à medida que eles surgem, mas também a antecipação e prevenção de problemas futuros. Com isso, a pesquisa de marketing auxilia o tomador de decisão, apresentando fatos pertinentes, analisando-os e sugerindo possíveis ações de ordem prática. Atualmente, notamos que para cada problema enfrentado pelos profissionais de marketing existe um tipo de pesquisa mais apropriado que visa encontrar e/ou apresentar as soluções específicas. Deste modo, a metodologia de pesquisa qualitativa destaca-se como uma das muitas maneiras.

De acordo com Malhotra (2001, p. 155) a pesquisa qualitativa é definida como uma técnica de “...pesquisa não-estruturada, exploratória, baseada em pequenas amostras, que proporciona insights e compreensão do contexto do problema” que está sendo estudado. Outros autores, buscando serem mais claros e diretos, citam que seu objetivo é simplesmente compreender o que está na mente do consumidor (AAKER; KUMAR; DAY, 2001).

Segundo Freitas e Janissek (2000, p. 25) a análise qualitativa se baseia "na presença ou ausência de uma dada característica, [enquanto] a análise quantitativa busca identificar a freqüência dos temas, palavras, expressões ou símbolos considerados”. O seu foco na essência está em proporcionar melhor visão e 
compreensão do contexto em estudo, ou seja, dar maior familiaridade a um problema, enquanto a pesquisa quantitativa procura quantificar os dados e aplicase alguma forma da análise estatística (como, por exemplo, os softwares SPSS ${ }^{\circledR}$, SAS ${ }^{\circledR}$, Minitab ${ }^{\circledR}$, Excel ${ }^{\circledR}$ etc.) para tratamento dos dados. Por outro lado, o uso de técnicas e softwares de análise de dados em pesquisa qualitativa (e.g. www.sphinxbrasil.com) é um tema de crescente interesse e importância no campo da Gestão e da Informação, não sendo mais de exclusividade das pesquisas quantitativas (FREITAS; JANISSEK, 2000).

Para alguns pesquisadores (CAHILL, 1998) existem várias razões para se utilizar essa pesquisa qualitativa em marketing, como, por exemplo: alcançar a compreensão de determinadas razões, determinar o grau de preferência dos consumidores em relação a marcas concorrentes, descobrir motivações subjacentes, desenvolver uma compreensão inicial de um problema, dentre outras.

Porém um problema muito encontrado, quando se executa a pesquisa, além de ser ressaltado por alguns autores (DAY, 1997; GABRIEL, 1990) como muito perigoso para o êxito da organização, deve ser abordado. Assim, os resultados obtidos na pesquisa qualitativa são usados incorretamente, quando considerados conclusivos e utilizados para fazer generalizações em relação à populaçãoalvo; assim, o ideal é a utilização de pesquisas quantitativas como complemento (FREITAS; CUNHA; MOSCAROLA, 1996; MARCHETTI, 1995), buscandose generalizações dos resultados, ao invés de usá-las mutuamente como concorrentes (HUNT, 1991). Todavia a pesquisa qualitativa possui variações em sua formação, conhecidas como técnicas. No próximo tópico discutiremos especificamente seis destas técnicas de pesquisa.

\section{As Variações das Técnicas de Pesquisa Qualitativa Aplicada em Marketing}

Com a crescente proliferação dos problemas mercadológicos, o método de pesquisa qualitativa em marketing foi ganhando seu espaço dentre os pesquisadores e posteriormente sendo dividida em diferentes técnicas. No Encontro Nacional de Programas de Pós-Graduação em Administração (EnAnpad - área Marketing), por exemplo, de 1990 a 1999, 32\% dos artigos apresentados empregavam diferentes métodos de pesquisa qualitativa (PERIN et al., 2000).

Assim, dentre essas diferentes variações, o nosso objeto de análise são as mais utilizadas no contexto da administração mercadológica, a saber: (a) 
Entrevistas em Profundidade, (b) Grupos de Foco, (c) Técnicas Projetivas, (d) Observação, (e) Zaltman Metaphor Elicitation Technique (ZMET), (f) Autodriving e (g) Filmes. Apesar de essas técnicas de pesquisa terem diferenças em suas composições, todas têm raízes nos aspectos psicanalíticos e clínicos da psicologia e algumas técnicas enfatizam perguntas abertas e diretas (AAKER; KUMAR; DAY, 2001; MATTAR, 1999) para estimular os entrevistados a revelarem seus pensamentos e crenças mais profundas. A seguir debateremos cada técnica individualmente.

\section{Entrevistas de Profundidade}

Pode se dizer que a entrevista de profundidade (in-depth) é definida como uma entrevista não-estruturada, direta, pessoal, em que um respondente de cada vez é instado por um entrevistador altamente qualificado a revelar motivações, crenças, atitudes e sentimentos sobre determinado tópico (NOTESS, 1996).

Neste processo o entrevistador inicia com uma pergunta genérica, e posteriormente incentiva o entrevistado a falar livremente sobre o tema (ex. O senhor poderia falar sobre fazer compras no Supermercado Carrefour?). Por sua vez a duração pode variar de 30 a 60 minutos, embora existam casos especiais que podem levar até mesmo horas, dada a natureza do problema (CHAUVEL, 2000).

Quando aplicada, a entrevista individual pode ser classificada em três categorias distintas, em função do grau de estruturação do guia de entrevista ${ }^{(3)}$ utilizado pelo entrevistador (DELBÈS; GRAMONT, 1983 apud MARCHETTI, 1995). A saber: entrevista não-estruturada; entrevista semi-estruturada e entrevista estruturada.

O fator comum nestas três categorias de guia encontra-se em incorporar perguntas abertas, permitindo a quem está respondendo fazê-lo a partir de suas opiniões e motivações. Devido a esse tipo de questão, as perguntas são mais reveladoras, pois não se limitam às respostas dos entrevistados (KOTLER, 2000), como é costume na investigação quantitativa.

É necessário que o pesquisador reconheça que nem sempre é conveniente utilizar métodos plenamente estruturados (formais) para obter informações dos respondentes. Algumas pessoas podem não querer responder a certas perguntas; ou, mesmo que sejam incapazes disso, o seu comportamento é influenciado por fatores de que eles não têm consciência ou que eles simplesmente não queiram mencionar diretamente em razão de certos mecanismos de defesa (DICHTER, 1964 apud MARCHETTI, 1995). Além do mais, é importante que o pesquisador 
não somente deixe o entrevistado o mais livre possível para expressar suas opiniões, como também possua conhecimentos de psicologia e lingüística (ou contratar profissionais com tal gabarito) para a análise dos dados.

\section{Focus Group}

O grupo de foco (focus group) teve origem na sociologia. Hoje, é amplamente utilizado na área de marketing e também tem crescido em popularidade em outros campos de ação (OLIVEIRA; FREITAS, 1998).

É uma entrevista realizada por um moderador bem treinado de uma forma nãoestruturada e natural, com um pequeno grupo de respondentes visando descobrir "possíveis idéias ou soluções para um problema de marketing por meio da discussão do tema” (AAKER; KUMAR; DAY, 2001, p. 211).

O focus group constitui-se na técnica mais importante de pesquisa qualitativa. Profissionais como Garee e Schori (1997) que executam a pesquisa de marketing constantemente consideram esta técnica um sinônimo da pesquisa qualitativa. Oliveira e Freitas (1998, p. 85) salientam que o seu objetivo é “...obter entendimento dos participantes sobre o tópico de interesse da pesquisa, não importando se é utilizado sozinho ou em conjunto com outros métodos, nem mesmo se busca questões ou respostas”.

Os grupos geralmente são constituídos de 08 a 12 entrevistados que se reúnem com um moderador ${ }^{(4)}$ para um debate em grupo, focando um produto ou qualquer outro assunto de interesse da pesquisa. Estas reuniões são geralmente registradas, utilizando-se aparelhos como câmaras de TV, máquinas fotográficas, etc. e, em sua maioria, observadas através de um vidro que permite ver, sem ser visto, conhecido como one-way mirror.

No focus group os entrevistados são estimulados a debater seus interesses, atitudes, reações, motivos de estilo de vida, sentimentos acerca da categoria de produtos, experiência de uso e os moderadores vão registrando as considerações feitas pelos participantes. É importante salientar que a chave para o seu sucesso está nas livres intervenções e opiniões de seus membros, o que, no entanto, demanda habilidade do moderador para fazer com que todos participem da discussão, sem que se crie nenhum tipo de ascendência em dinâmica de grupo (ROSSI; SLONGO, 1997).

Nelson e Fronteazak (1988, p. 41-42) comentam a importância de formar os grupos por pessoas com características “...semelhantes e com interesses convergentes...” em relação ao problema analisado, pois “...de nada adiantaria 
reunir um grupo de jovens solteiros sem filhos para discutir sobre os atributos desejados em fraldas descartáveis”.

Os grupos de foco podem apresentar inúmeras variações do processo padrão de execução (ver mais em OLIVEIRA; FREITAS, 1998), as mais conhecidas e utilizadas técnicas são: grupo de foco de duas vias; grupo de foco individual; grupo moderador dual; grupo duelador $\Leftrightarrow$ moderador; grupo respondente $\Leftrightarrow$ moderador; grupo cliente $\Leftrightarrow$ participante; grupo minigrupos; grupo de telesessão (HARTLEY; PROUGH; FLASCHNER, 1983, p. 244-245); e grupo on-line $e^{(5)}$ para pesquisa internacional (PARKS, 1997).

Devido à rapidez com que o focus group gera informações, o pesquisador deve estar preparado para registrar as falas dos participantes, o que pode ser feito com uso de gravadores ou taquigrafia. Outros pontos importantes para o pesquisador são os seguintes: saber conduzir a discussão do grupo e não apenas deixar um entrevistado falar mais do que os demais no grupo; elaborar uma sala de reunião que gere liberdade aos entrevistados; e reconhecer as linguagens escritas em ambientes on-line $e^{(6)}$.

Em resumo, podemos dizer que o focus group é uma técnica na pesquisa qualitativa, cuja aplicação é muito útil (KOTLER, 2000), principalmente nas ciências sociais, além de ser amplamente utilizada em áreas como Gestão, Marketing, Decisão e Sistemas de Informação, dentre outras. Algumas de suas características gerais podem ser destacadas, como a integração de pessoas, a seqüência de sessões, a obtenção de dados qualitativos e o foco em um tópico, além de essa variação poder ser utilizado isoladamente ou associada a outras técnicas, o que permite reforçar um desenho de pesquisa (OLIVEIRA; FREITAS, 1998).

\section{Técnicas Projetivas}

Muito utilizada em 1940-50, durante o interesse na pesquisa motivacional ${ }^{(7)}$, as técnicas projetivas (projective techniques) partem de um princípio bastante simples, por meio de um estímulo o indivíduo projeta seus aspectos subjetivos, atitudes, comportamento, opiniões, etc., o que, por alguma razão, não faria espontaneamente (PERRIEN; EMMANUEL; ZINS, 1984 apud MARCHETTI, 1995). Elas são planejadas para acessar os motivos fundamentais dos indivíduos, apesar das racionalizações inconscientes ou dos esforços de encobrimento consciente (SCHIFFMAN; KANUK, 2000).

Malhotra (2001, p. 165) define a técnica projetiva como "uma forma nãoestruturada e indireta de perguntar que incentiva os entrevistados a projetarem 
suas motivações, crenças, atitudes ou sensações subjacentes sobre os problemas em estudo”.

A utilidade dessa técnica é evidenciada quando se observam as seguintes diretrizes:

1. As técnicas projetivas devem ser utilizadas quando a informação desejada não pode ser obtida com precisão por métodos diretos.

2. As técnicas projetivas devem ser usadas para pesquisa exploratória, para proporcionar entendimento e compreensão iniciais.

3. Em vista de sua complexidade, as técnicas projetivas não devem ser usadas ingenuamente.

Um executivo que acabara de adquirir um belo automóvel para obter mais status na sociedade, em uma pesquisa normal pode responder que buscou o automóvel simplesmente porque gostou, para não ficar constrangido. Com a utilização da técnica projetiva é possível descobrir a verdadeira causa desta compra (KOTLER, 2000).

Para Marchetti (1995, p. 21) mesmo aparentando “...simplicidade, a utilização das técnicas projetivas em marketing deve ser feita com bastante cuidado”, pois as dificuldades de se avaliar a validade e a confiabilidade dos testes projetivos podem conduzir a resultados distorcidos.

Assim, o pesquisador deve possuir formação especializada e aptidão para conduzir as entrevistas e boa capacidade de raciocínio para gerar os debates. Além do mais espera-se que saiba decidir entre os estímulos mais adequados (fotos, objetos, sons) a serem utilizados na entrevista. Outro ponto importante está na análise dos dados. Utilização de antropólogos, psicólogos, sociólogos, terapeutas etc. é fortemente recomendável ao pesquisador na técnica projetiva.

\section{Observação}

Melhor do que interagindo com os respondentes verbalmente, os pesquisadores podem simplesmente observar como os consumidores se comportam durante a compra, como fazem as decisões sobre os produtos na loja, ou como consomem estes produtos em suas casas (NELEMS, 2001). Na pesquisa por observação, o pesquisador efetua um registro sistemático de dados, de comportamentos, de fatos e de ações, a fim de obter subsídios sobre determinado fenômeno que está sendo estudado. 
Na Antropologia, por exemplo, essa técnica é de essencial relevância, uma vez que busca constatar diferenças entre costumes e hábitos culturais. A observação não consiste apenas em ver e ouvir seu objeto de estudo, mas também em examinar fatos ou fenômenos.

Kurcina (2000) salienta um exemplo muito importante dessa técnica no ramo empresarial. De acordo com um estudo, uma empresa estava constatando redução em seus níveis de vendas e satisfação do consumidor. Uma observação, com o auxílio de vídeos combinada com entrevistas pessoais, revelou que os compradores estavam confusos com as informações contidas na embalagem do produto, escolhendo assim os produtos errados para suas necessidades e ficando naturalmente insatisfeitos. Detectada a falha a empresa acabou alterando as informações de suas embalagens, recuperando, assim, as vendas e os níveis de satisfação anteriores.

A pesquisa observacional é uma técnica importante de pesquisa do consumidor. As empresas reconhecem que o melhor modo de se obter um entendimento profundo do relacionamento entre as pessoas e os produtos ocorre por meio da observação de seu processo de compra e de uso dos produtos (SCHIFFMAN; KANUK, 2000). Com o uso dessa técnica, o pesquisador poderá, por exemplo, verificar (naturalmente) como o usuário realmente interage com o produto na hora da compra. Dentre as variações possíveis da pesquisa observacional, podemos destacar algumas muito utilizadas, tais como a natural e a $\operatorname{artificial~}^{(8)}$.

Um cuidado especial está na obtenção dos dados. Pesquisadores deverão ser aptos e bem treinados para utilizar as técnicas de coleta de dados disponíveis na observação, tais como: psicogalvanômetro, contadores de tráfico, pupilômetro, intensidade de voz etc ${ }^{(9)}$.

\section{ZMET - Zaltman Metaphor Elicitation Technique ${ }^{(10)}$}

Identificar o que o cérebro cria, quando este possui familiaridade com o produto, suas idéias, percepções, atitudes e preferências em relação a ele, verificar quais são as ligações entre determinadas variáveis que uma marca proporciona e desvendar os desafios traçados entre corpo, mente, processos psicológicos e sociedade são novos problemas mercadológicos que surgem na vida do consumidor (ZALTMAN, 2000). Saber como recuperá-los, decodificá-los, interpretá-los e apresentá-los são algumas das respostas que a técnica Zaltman Metaphor Elicitation Technique (ZMET) pode proporcionar. 
A ZMET é uma técnica qualitativa de caráter híbrido que direciona os entrevistados a coletar e edificar metáforas (imagens figurativas que representam alguma outra coisa) com produtos ou compras, baseados na técnica projetiva (KRAFT; NIQUE, 2002). Ela faz uso de gravações fotográficas e áudio para enriquecer as entrevistas normais.

Segundo seu criador, o professor Gerald Zaltman (1997, p. 428) da Harvard Business School, a técnica ZMET “envolve uma intensa exploração da geração própria de metáforas por parte dos administradores e consumidores”. Sintetizando, “...ela revela o comportamento do consumidor não só ouvindo a sua voz, mas também vendo a voz do cliente” (SHETH; MITTAL; NEWMAN, 2001, p. 129).

Áreas que aparentemente não têm nada a ver com o estudo do comportamento do consumidor como: neurociência cognitiva, neurobiologia, psicolingüística, sociologia visual, antropologia visual, semiótica, fototerapia, artes foram pesquisadas para delinear as premissas teóricas da ZMET (KRAFT; NIQUE, 2002).

Na verdade, essa técnica pode ser utilizada por pesquisadores do consumidor como maneira para enriquecer o corpo dos dados qualitativos, providenciando uma perspectiva de ação (GOULD et al., 1974 apud HEISLEY; LEVY, 1991). Outra utilização da ZMET concentra-se na construção de um mapa de consenso dos pensamentos do entrevistado (ver COULTER; ZALTMAN, 1994). Esse mapa de consenso fornece uma visualização mais entendível dos construtos utilizados pelo entrevistado. Indo além, a ZMET providencia fundamental compreensão dos consumidores pela identificação de um conjunto de significados em vários níveis de experiência. Isso ajuda a compreender como o inconsciente e o consciente trabalham juntos em um processo de interação para: (1) criar necessidades; (2) influenciar o critério pela satisfação deles; (3) formar a experiência da satisfação das necessidades; e (4) expandir os julgamentos sobre essas experiências (ZALTMAN; OLSON) ${ }^{(11)}$.

A execução da Zaltman Metaphor Elicitation Technique não é uma obra fácil. Na realidade um conhecimento das premissas teóricas que envolvem a técnica é fundamental para sua boa execução e validade. Kraft e Nique (2002, p. 4) salientam que cada etapa de execução deve ser “...cuidadosamente projetada para evocar aspectos racionais e emocionais dos participantes”. Na Tabela 1 apresentamos os principais passos para a execução da técnica ZMET. 


\section{Tabela 1: Principais Passos para a Execução da Técnica ZMET}

\begin{tabular}{|c|c|c|}
\hline Passos & Nome & Descrição \\
\hline Passo 01 & Narração & $\begin{array}{l}\text { Consumidor descreve o conteúdo de cada fotografia que é exposta para } \\
\text { ele. }\end{array}$ \\
\hline Passo 02 & Imagens Perdidas & $\begin{array}{l}\text { Consumidor descreve as fotografias que ele não estava apto para obter ou } \\
\text { explicar suas relevâncias. }\end{array}$ \\
\hline Passo 03 & Tarefas em Comum & Tarefas em Comum. Consumidor ordena as suas fotos em significados. \\
\hline Passo 04 & $\begin{array}{l}\text { Obtenção do } \\
\text { Construto }\end{array}$ & $\begin{array}{l}\text { Uma versão modificada da técnica Repertory Grid e da técnica } \\
\text { Laddering }{ }^{(12)} \text { usadas para obter os construtos e suas relações. As figuras } \\
\text { dos consumidores servem como estímulos. }\end{array}$ \\
\hline Passo 05 & $\begin{array}{l}\text { Figura Mais } \\
\text { Representativa }\end{array}$ & Consumidor indica a figura mais representativa. \\
\hline Passo 06 & Imagem Oposta & $\begin{array}{l}\text { Consumidor descreve figuras que representam o oposto de sua tarefa, ou } \\
\text { seja, o que não é tal coisa. }\end{array}$ \\
\hline Passo 07 & Imagem Sensorial & $\begin{array}{l}\text { Descrições são obtidas do que é, e do que não é, do gosto, do cheiro, do } \\
\text { sabor, do som, das cores e emoções dos conceitos explorados. }\end{array}$ \\
\hline Passo 08 & O Mapa Mental & $\begin{array}{l}\text { O Mapa Mental. Consumidor cria um mapa, usando os construtos que ele } \\
\text { tem obtido do passo } 7 \text {. }\end{array}$ \\
\hline Passo 09 & Imagem Resumida & $\begin{array}{l}\text { O consumidor, com a assistência de um técnico, cria uma imagem } \\
\text { resumida, utilizando técnicas de imagens digitais. }\end{array}$ \\
\hline Passo 10 & $\begin{array}{l}\text { Mapa de Consenso } \\
\text { Final }\end{array}$ & $\begin{array}{l}\text { Pesquisadores criam um mapa ou um modelo casual, envolvendo os mais } \\
\text { importantes construtos. }\end{array}$ \\
\hline
\end{tabular}

Fonte: adaptado de Coulter e Zaltman (1994, p. 503).

\section{Pesquisa Visual: Autodriving}

Para Heisley e Levy (1991, p. 258-259) “a pesquisa com a utilização de fotos não é nova, ou seja, em 1894 Fort Rupert usou fotografias de máscaras e rituais para entrevistar informantes e estudar dança e movimento; em 1949 Gregory Bateson e Margaret Mead utilizaram fotos e textos em conjunto para providenciar um entendimento de uma determinada cultura; em 1921 Robert Flaherty utilizouse da câmera participante, ou seja, ele morou com seus informantes, os esquimós, e por oito anos os envolveu em um filme; após isso, os esquimós assistiram ao filme e fizeram ativos comentários sobre seus próprios comportamentos e em 1983 a primeira conferência em sociologia visual tomou corpo, The Visual Sociology Review".

A pesquisa visual conquistou momentos de naturalidade na pesquisa qualitativa de marketing pela sua eficiência em explicar fatores subjetivos do consumidor. Deste modo em 1995 a Association for Consumer Research(ACR, www.acrweb.org ) introduziu uma sessão especial de pesquisa visual no comportamento do consumidor, provendo uma introdução à técnica de fotografia em vídeo como uma metodologia de pesquisa (HEISLEY; LEVY, 1991).

Autodriving, como também é chamada a pesquisa visual, pode ser utilizada por pesquisadores do consumidor como maneira de enriquecer o corpo dos dados qualitativos para providenciar uma perspectiva de ação (GOULD et al., 1974 
apud HEISLEY; LEVY, 1991). Na verdade, a técnica possui caráter diferenciado, porquanto, por meio da foto o entrevistado projeta significados que ele encontra na foto para seu entrevistador e permite, em certa parte, fazer com que eles mesmos (os entrevistados) se entrevistem. Na verdade, não apenas fotografias podem ser utilizadas para a obtenção de dados, mas filmes e vídeos.

Para compreender melhor a técnica autodriving Heisley e Levy (1991) aplicaram-na em três famílias de classe média nos EUA. Neste estudo os autores buscaram compreender melhor o momento do jantar de uma família (consumo de comida). Primeiro, eles tiraram fotos da preparação e do consumo da janta pelas famílias (aprox. um total de 180 fotos). Segundo, eles entrevistaram os membros ( $1^{\text {a }}$ gravação [audio-taped]), utilizando as fotos (variando de 10 a 17 fotos mostradas), obtendo interessantes resultados; por fim, um terceiro momento aconteceu, quando os pesquisadores mostraram as fotos novamente acompanhadas das gravações da primeira parte (fizeram então a $2^{\mathrm{a}}$ gravação [audio-taped]). As entrevistas foram transcritas e os resultados ficaram associados em três áreas: associação de produtos; papéis de relação e reatividade. Por exemplo, em papéis de relação os pesquisadores identificaram que o pai tem como função entreter os filhos no momento da janta, ou seja, sua função é distribuir a comida e educá-los no processo de consumo (ver Figura 1).

\section{Figura 1: Consumo em Família}

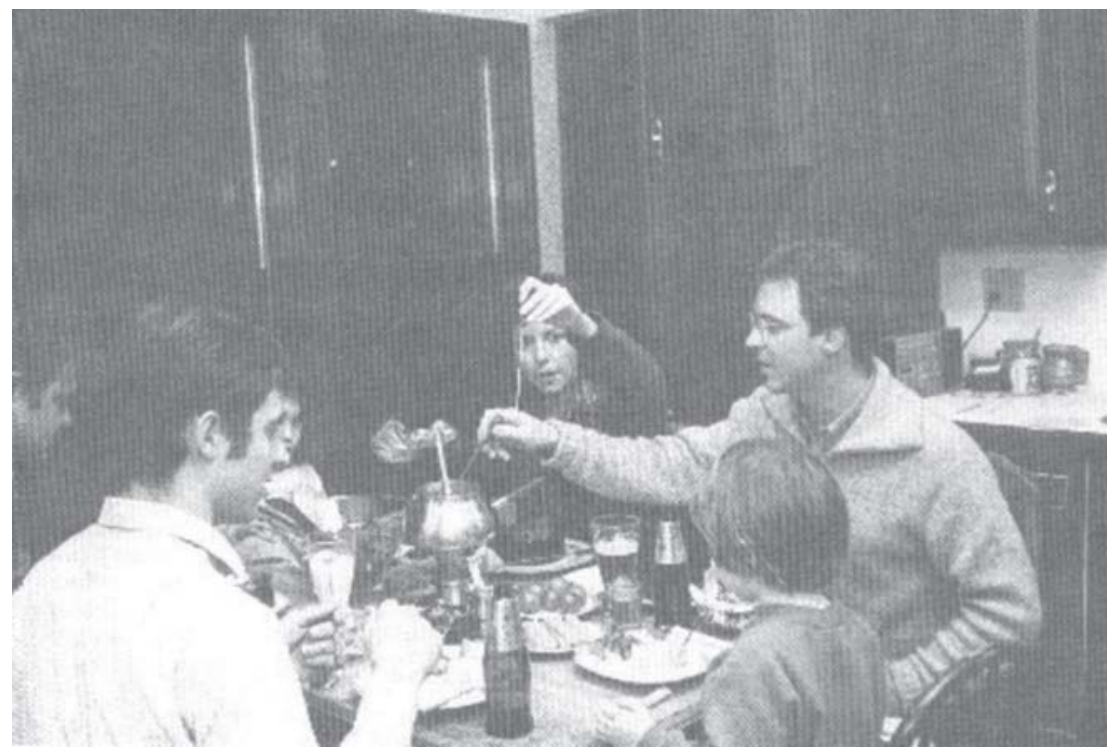

Fonte: Heisley e Levy, 1991, p.264. 
Holbrook (1987) também fez uso da técnica autodriving em sua pesquisa. A pesquisadora procurou identificar formas e tipos de consumo fanático de objetos (neste caso Discos de Vinil de Jazz). Um dos entrevistados de sua amostra fora ela mesmo, ou seja, a pesquisadora tirou fotos de seus materiais e perguntou (com o auxílio das fotos) o que eles significavam para ela mesma. Além do mais, para enriquecer os dados de sua pesquisa, ela utilizou-se de músicas como estímulo para as respostas.

No ano seguinte Holbrook (1988) buscou mensurar o papel da interpretação psicanalítica do consumo. Especificamente o trabalho deu foco na própria coleção de objetos de arte da pesquisadora. Além de utilizar-se de entrevistas de profundidade, video-tape, gravações, fotos, e outros métodos multifacetados de obtenção de dados, a sua experiência como informante indicou alguns significados latentes, que podem estar muito mais escondidos. Assim, a idéia do artigo também foi levantar importantes questões, onde o meio naturalístico para e o psicanalítico começa (HOLBROOK, 1988).

Porém, infelizmente, existem algumas características pelas quais os profissionais rejeitam a pesquisa visual (HEISLEY, 2001), como as que vêm explicitadas abaixo.

1. Os profissionais acreditam que o trabalho de uma pesquisa visual pode ser visto como menos sério pela academia.

2. Um entendimento visual é acessível. Isto permite que o leitor, neste caso a pessoa que está vendo as fotos, possua muitas interpretações. Esta perda de controle pelo entrevistador pode ser desconfortável e ameaçadora.

3. Em comportamento do consumidor os pesquisadores podem sentir falta de familiaridade com a alta qualidade e com o vídeo como produto.

4. Scholars são influenciados pelo viés geral da população, que pode ser mais intelectual que visual.

5. Não existe um processo peer review para legitimar o trabalho.

6. Este processo é muito trabalhoso.

Deste modo, cuidados especiais na execução do autodriving são fundamentais para a validade da investigação. Pesquisadores devem (a) reconhecer as máquinas fotográficas e materiais ideais para tirarem as fotos; (b) cuidar para não deixar os entrevistados inibidos no momento em que as fotos estão sendo tiradas (Figura 1); e (c) saber interpretar os múltiplos significados que as fotos representam (BALL; SMITH, 1992). Pré-testes em 
amostras pequenas poderiam ser bons começos para a execução e estimular a familiarização com a técnica.

Em resumo, compreendemos que a pesquisa com fotos é um novo segmento que necessita de maiores esclarecimentos e investigações no cenário brasileiro. Além de revelar dados interessantes, a técnica carece de maiores refinamentos e questionamentos sobre validade e aplicabilidade.

\section{Filmes}

Em sua segunda edição, ocorrida em 2003, o Festival de Filmes da ACR apresentou treze filmes, demonstrando uma nova oportunidade para exploração de dados qualitativos e experimentais em pesquisas de mercado. Alguns dos trabalhos apresentados foram filmes visando apresentar: (i) a transformação das pessoas ou da comunidade de um pequeno vilarejo grego para um destino turístico; (ii) o que representa um lar para seu dono, dentre outros aspectos (ver ALLISON, 2003).

É interessante que no procedimento de pesquisa por filmes é possível ver e ouvir ao mesmo tempo; assim o pesquisador pode fazer as interrogações e gravar os dados ele mesmo; mas deve haver cuidados na condução e execução do autodriving, como nas outras técnicas.

Os outros trabalhos visuais produzidos neste congresso não serão discutidos detalhadamente neste artigo por questão de espaço. Todavia fica como indicação a visita ao site da associação para maiores consultas.

Acreditamos que a utilização de filmes pode ser compreendida como forma mais avançada da técnica autodriving. A montagem e elaboração, tanto quanto a análise, requerem forte e minucioso cuidado; mas o resultado final do material pode levantar informações valiosas para a investigação. Embora possua diversos problemas, tais como questões éticas, qualidade do material final etc., a filmagem pode ser interessante alternativa para pesquisas qualitativas no cenário nacional.

Em resumo, cada técnica tem características únicas em seu ventre e podem ser utilizadas para uma ampla gama de objetivos. Subvariações de cada uma, modos de aplicação e outros aspectos não foram discutidos detalhadamente neste artigo por questão de espaço. No próximo tópico os pontos positivos e negativos de cada uma delas serão discutidos. 


\section{Uma Revisão Ampliada das Técnicas}

Uma vez explicadas e citadas as técnicas mais utilizadas nas pesquisas qualitativas em marketing, a apresentação das vantagens e desvantagens relativas de cada uma se faz necessária, à medida que revelam aos pesquisadores seus benefícios e deficiências (Tabela 2).

\section{Tabela 2: As Técnicas de Pesquisa Qualitativa e suas Vantagens/Desvantagens}

\begin{tabular}{|c|c|c|}
\hline MÉTODO & VANTAGEM & DESVANTAGEM \\
\hline $\begin{array}{l}\text { Entrevista } \\
\text { Profundidade }\end{array}$ & $\begin{array}{l}\text { - } \begin{array}{l}\text { Resposta isenta da influência do grupo, } \\
\text { permitindo uma maior autenticidade. } \\
\text { - } \quad \text { A informação é mais completa e detalhada. } \\
\text { Coloca em evidência o processo de } \\
\text { decisão do consumidor. } \\
\text { - É flexível, permitindo modificações no } \\
\text { processo de entrevista. } \\
\text { A entrevista individual é mais adaptada às } \\
\text { técnicas projetivas que as demais formas } \\
\text { de coleta de informações. } \\
\text { Informações confidenciais são mais fáceis } \\
\text { de se obter. } \\
\text { - Permite a coleta de informações onde as } \\
\text { reuniões de grupo são realizadas com } \\
\text { maior grau de dificuldade, como no caso } \\
\text { das reuniões com empresários } \\
\text { concorrentes. }\end{array} \\
\end{array}$ & $\begin{array}{l}\text { - Demanda entrevistadores habilidosos e } \\
\text { capazes de fazer boas entrevistas, que em } \\
\text { sua maioria são caros. } \\
\text { - } \begin{array}{l}\text { A falta de estrutura pode tornar os } \\
\text { resultados suscetíveis à influência do } \\
\text { entrevistador. }\end{array} \\
\text { - A qualidade e completude dos resultados } \\
\text { dependem pesadamente da habilidade do } \\
\text { entrevistador. } \\
\text { - Grande dificuldade de analisar e } \\
\text { interpretar os dados obtidos, sendo } \\
\text { normalmente necessária a intervenção de } \\
\text { psicólogos competentes. } \\
\text { Altos custos no geral. }\end{array}$ \\
\hline Focus Group & 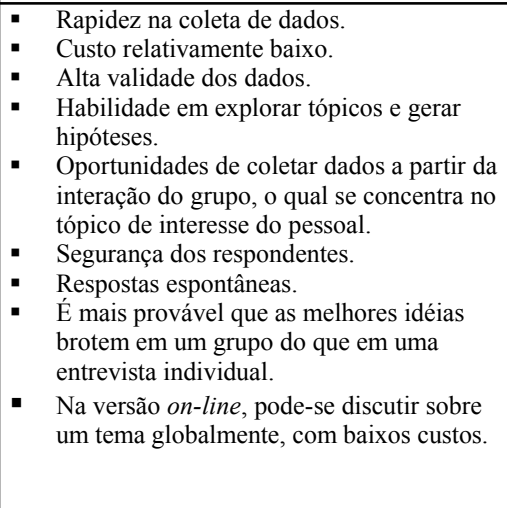 & $\begin{array}{l}\text { - Possibilidades da opinião do grupo ser } \\
\text { afetada pela intervenção de pessoas que } \\
\text { falam muito. } \\
\text { - } \quad \text { Não é baseado em um ambiente real. } \\
\text { Pesquisador tem menor controle sobre os } \\
\text { dados gerados. } \\
\text { - Não é possível saber se a interação em } \\
\text { grupo reflete ou não o comportamento } \\
\text { individual. } \\
\text { - Os dados são mais difíceis de analisar. } \\
\text { Exige entrevistadores treinados } \\
\text { cuidadosamente. } \\
\text { A discussão deve ser conduzida em } \\
\text { ambiente que propicie o diálogo. } \\
\text { - Na Internet o entrevistador não sabe quem } \\
\text { o respondente é, e se tem capacidade para } \\
\text { falar do tema. }\end{array}$ \\
\hline $\begin{array}{l}\text { Técnicas } \\
\text { Projetivas }\end{array}$ & $\begin{array}{l}\text { - } \begin{array}{l}\text { Provoca uma resposta que o indivíduo não } \\
\text { daria ou não poderia dar se soubesse } \\
\text { objetivo de estudo. }\end{array} \\
\text { - } \begin{array}{l}\text { Muito proveitosa quando p/ motivações, } \\
\text { crenças e atitudes estão agindo em um } \\
\text { nível subconsciente. }\end{array} \\
\text { - } \begin{array}{l}\text { Fornece as verdadeiras razões de um } \\
\text { determinado problema. }\end{array}\end{array}$ & $\begin{array}{l}\text { Dificuldade de se avaliar a validade e a } \\
\text { confiabilidade dos testes. } \\
\text { - } \quad \begin{array}{l}\text { Exige um entrevistador 'altamente' } \\
\text { treinado e intérpretes qualificados para } \\
\text { analisar as respostas. }\end{array} \\
\text { - Existe um sério risco de tendenciosidade } \\
\text { na interpretação. } \\
\text { - Exige do entrevistado (em alguns casos) } \\
\text { um comportamento não usual. }\end{array}$ \\
\hline
\end{tabular}




\section{(conclusão)}

\section{Tabela 2: As Técnicas de Pesquisa Qualitativa e suas Vantagens/Desvantagens}

\begin{tabular}{|c|c|c|}
\hline Observação & $\begin{array}{l}\text { Não há tendenciosidade de relato, e a } \\
\text { tendenciosidade potencial causada pelo } \\
\text { entrevistador e pelo processo de entrevista } \\
\text { é eliminada ou reduzida. } \\
\text { Obter certos tipos específicos de dados } \\
\text { (aqueles que até mesmo o respondente } \\
\text { desconhece, ou não pode comunicar). } \\
\text { Exige menos do observador do que outras } \\
\text { técnicas. } \\
\text { Custo baixo em determinados casos pelo } \\
\text { fato do fenômeno observado durar } \\
\text { relativamente pouco. } \\
\text { Grande capacidade de observação em um } \\
\text { contexto natural. } \\
\text { Amplamente utilizada para entender o } \\
\text { processo de compra e de consumo. }\end{array}$ & $\begin{array}{l}\text { As razões de comportamento observado } \\
\text { podem não ser determinadas em alguns } \\
\text { casos. } \\
\text { Alguns casos observáveis podem ser } \\
\text { antiéticos, ou em casos particulares, pode } \\
\text { ter-se a dificuldade de coleta de dados. } \\
\text { Demanda tempo e excelentes profissionais } \\
\text { na análise de dados. } \\
\text { - Se a observação não for camuflada o } \\
\text { entrevistado pode induzir o pesquisador } \\
\text { ao erro propositalmente. } \\
\text { O observado tende a criar impressões } \\
\text { favoráveis ou desfavoráveis no } \\
\text { observador. } \\
\text { Fatores imprevistos podem interferir na } \\
\text { tarefa do pesquisador. }\end{array}$ \\
\hline$\overline{\text { ZMET }}$ & $\begin{array}{l}\text { Alavanca o volume de dados qualitativos } \\
\text { providenciando uma perspectiva de ação. } \\
\text { Possibilidade de visualização de dados } \\
\text { familiares pelo entrevistado de modos } \\
\text { pouco conhecidos. } \\
\text { Obtenção de um mapa de consenso que } \\
\text { normalmente contém entre } 20 \text { e } 30 \\
\text { constructos interligados. } \\
\text { Dirige os entrevistados para uma entrevista } \\
\text { guiada por eles próprios proporcionando } \\
\text { uma maior facilidade do entrevistador } \\
\text { ver/receber suas opiniões. } \\
\text { Identifica a informação não-verbal, onde } \\
\text { representa } 1 / 3 \text { de toda o significado social } \\
\text { (ZALTMAN, 1996). }\end{array}$ & $\begin{array}{l}\text { - } \begin{array}{l}\text { Determinadas fotografias podem não } \\
\text { refletir o mesmo costume, hábito em } \\
\text { pesquisa internacional. }\end{array} \\
\text { Dificuldade de encontrar/elaborar as fotos } \\
\text { que realmente significam aquilo que se } \\
\text { pretende mostrar. } \\
\text { - Não reconhecer o número de fotos ideal } \\
\text { para ser demonstrada na sessão, tornando- } \\
\text { a tediosa. } \\
\text { - Demanda muito tempo. } \\
\text { Altos custos financeiros. }\end{array}$ \\
\hline Autodriving & $\begin{array}{l}\text { - Ajuda a eliminar o stress de uma entrevista } \\
\text { por fazer o indivíduo não ser 'somente' o } \\
\text { sujeito da entrevista. } \\
\text { - } \quad \text { Ajuda a ilustrar os insigths e sentimentos } \\
\text { dos entrevistados. } \\
\text { - Revela dados mais profundos. } \\
\text { - Providencia uma forma de ckeck-in para o } \\
\text { pesquisador aumentar a credibilidade da } \\
\text { interpretação da pesquisa. } \\
\text { - Providenciou (naquele estudo) os } \\
\text { entrevistados a descobrirem e expressarem } \\
\text { o poder e conflitos de relações que estão } \\
\text { implícitas em suas realidades diárias. }\end{array}$ & 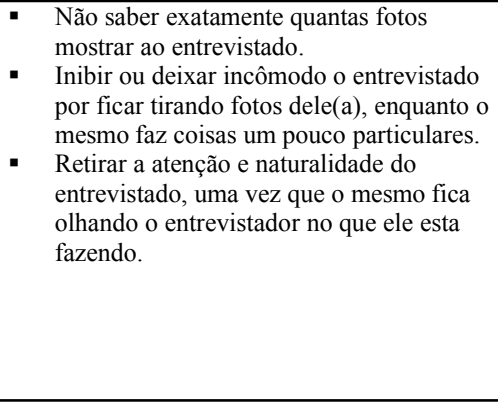 \\
\hline Filmes & $\begin{array}{l}\text { Interação - possibilidade do entrevistado } \\
\text { brincar e gerar mais idéias por discutir } \\
\text { interativamente com o pesquisador. }\end{array}$ & $\begin{array}{l}\text { - } \quad \text { Inibir ou deixar incômodo o entrevistado. } \\
\text { - } \quad \text { Pão produzir dados válidos. } \\
\text { Por ficar tirando fotos dele(a), enquanto } \\
\text { o mesmo faz coisas um pouco } \\
\text { particulares. }\end{array}$ \\
\hline
\end{tabular}

Fonte: autores.

Trabalhos de comparação entre técnicas qualitativas são importantes para a pesquisa de mercado (CATTERALL, 1998). Assim, é importante frisar que o pesquisador deve ser o conhecedor das técnicas e de seu poder de fogo; mas 
uma utilização errônea da mesma, ou mesmo a utilização de uma técnica que não condiz com a realidade do problema, pode levar, e muitas vezes leva, todo um projeto por água abaixo.

\section{Considerações Finais}

A pesquisa qualitativa veio consolidando-se ao longo da história científica como prática que possibilita o desvendamento de objetos subjetivos que, por sua complexidade, exigem compreensão mais aprofundada dos fenômenos que os envolvem. Em alguns casos, de nada adianta uma pesquisa quantitativa sem antes se ter as qualidades para serem quantificadas.

Neste artigo procurou-se detalhar as técnicas mais importantes da pesquisa qualitativa utilizadas em marketing, a saber: entrevistas de profundidade, focus group, técnicas projetivas, pesquisa observacional, ZMET, autodriving e filmes. No final, propomos uma tabela, incorporando as principais vantagens e desvantagens de cada uma destas. Acreditamos que o esclarecimento das técnicas possa estimular a utilização da metodologia de pesquisa qualitativa em nosso contexto, bem como alertar pesquisadores sobre seu respectivo emprego.

Profissionais de marketing, pesquisadores, institutos de pesquisas e aspirantes a pesquisadores poderão, com este trabalho, sanar dúvidas que altercam a utilização da pesquisa qualitativa em marketing e conhecer detalhadamente o conglomerado de suas características e variações.

Cabe ao responsável pela pesquisa definir qual técnica - dentre as várias apresentadas - é a mais apropriada para sua investigação. Não existe a melhor dentre elas, mas aquela mais apropriada para revelar, de forma acurada, o problema. A importância em se verificar a natureza do problema em questão, e os objetivos do projeto são essenciais, pois estes são fatores de suma importância para a escolha da técnica (FREITAS et al., 2000; ROLLER, 2001) e para o sucesso da investigação.

Vale lembrar que, ao realizar a pesquisa qualitativa, o pesquisador deve ser ético e respeitar o entrevistado, o que inclui a proteção do anonimato do participante e a manutenção de todas as afirmações e promessas feitas para assegurar sua participação. Outro ponto importante está no preparo do pesquisador; por exemplo, conhecimento das técnicas existentes, utilização de pré-testes, saber usar a ferramenta correta para coleta de dados, e ter ciência das ferramentas para análise dos dados são fundamentais para o sucesso da 
pesquisa. Além do mais, características como: práticas enganosas na pesquisa, invasão de privacidade, falta de preocupação com os assuntos do respondente, interesses pessoais e técnicas analíticas inapropriadas devem ser rigorosamente rejeitadas. Em resumo, há que se ter competência para analisar os dados posteriormente e, a partir da análise, extrair as relações que existem com situações típicas de marketing.

Sugestões para Pesquisas Futuras. Uma agenda para pesquisas futuras vem em boa hora no contexto nacional. Profissionais e pesquisadores, bem como professores poderão com essa agenda, desde que devidamente preparados, elaborar e/ou testar novas técnicas de pesquisas que estão sendo desenvolvidas no contexto internacional, ou até mesmo buscar verificar até onde vai, em termos de validade e confiabilidade, uma determinada variação.

É importante instigar a continuação de estudos das variações de técnicas de pesquisas qualitativas pelo fato de esse tópico encontrar-se em fase de amadurecimento no contexto nacional. Artigos que envolvem outras metodologias qualitativas tendem a chegar em bom momento, tanto para a academia quanto para a parte gerencial.

Algumas variações adicionais das técnicas de pesquisa qualitativa não foram comentadas nesse trabalho, não por uma questão de importância ou demérito, mas por questão de espaço físico, ficando como sugestões para desenvolvimento em investigações futuras; dentre eles destacamos:

. Técnicas qualitativas em pesquisas com crianças para o segmento infantil.

- Outros métodos de processamento das informações e técnicas de pesquisa para mensurar comunicação impressa (ver MCBRIDE, 2002).

- Mapas cognitivos (HINES, 2000). Uma técnica qualitativa desenhada para identificar causas e efeitos e explicar ligações casuais. Alguns pesquisadores utilizam-se dessa técnica para obterem construtos dos entrevistados e entendêlos melhor antes de executar a pesquisa em si.

. Novos estudos em algumas técnicas já existentes como a SSM - Soft Systems Methodology. Uma metodologia para análise, diagnóstico e modelagem de organizações ou situações para resolução de problemas (ver SOARES; COSENZA; GOMES, 2001).

. ZMET - Zaltman Metaphor Elicitation Technique, utilizando-se de muitos grupos em conjunto, ou times de administradores (ver ZALTMAN, 1996, 1997; 
ZALTMAN; COULTER, 1995); ou até mesmo pesquisas quantitativas, verificando a presença e forças das relações em atitudes e contexto de propagandas (COULTER; ZALTMAN; COULTER, 2001).

. Desenvolvimento e utilização da SEMAT - Semantic Attribution Test. Uma técnica de pesquisa utilizada para enriquecer os dados em grupos de foco (LEVINSON, 1999).

. KANO - Methodology. Uma técnica nova de pesquisa que emprega dados qualitativos e quantitativos. Essa técnica ajuda no processo de desenvolvimento do produto, reduzindo custos, e na diminuição do tempo de lançamento no mercado (GONÇALVES, 2000; GONÇALVES; GONÇALVES, 2001; NICITA; WALTERS, 2000).

. TAT - Thematic Apperception Test (ver mais em ROOK; LEVY, 1983 apud ENGLIS; SOLOMON, 2000).

- Autodriving. Utiliza-se de fotografias e vídeos em técnicas projetivas, principalmente em compras etnográficas e naturalísticas (ver ENGLIS; SOLOMON, 2000) $)^{(13)}$.

\section{Artigo recebido em 13.12.2003. Aprovado em 01.10.2004.}

\section{Notas}

${ }^{1}$ Os autores são extremamente gratos a Henrique Freitas, Raquel Janissek, Carlos Gomes, Sibila Kraft, Virginia Soares, e aos revisores anônimos da RAC pelos comentários concedidos, tão quanto a Sidney J. Levy e Gerald Zaltman pelos materiais adicionais encaminhados via correio.

${ }^{2}$ Revisão dos autores na RAC, RAE, RAUSP e EnAnpad.

${ }^{3}$ Definimos o guia de pesquisa como um roteiro que o entrevistador segue para executar a pesquisa.

${ }^{4}$ Em muitos casos o moderador também é o analista dos dados da pesquisa, em outros, o moderador não-analista é conhecido como moderador camuflado se fazendo de respondente para estimular e inibir a timidez dos respondentes.

${ }^{5}$ Pesquisas Internacionais on-line por vezes se utilizam de expressões atípicas, como por exemplo: :-) que significa feliz, :-| que significa neutro e :-( que significa triste.

${ }^{6}$ Principalmente em entrevistas com jovens que se comunicam por meio de expressões (vocabulário próprio deles) como: q, vc, heee, :), naummm, rs, bjs, []’s. 
${ }^{7}$ Em 1939, o psicanalista vienense Ernest Dichter começou a usar as técnicas psicanalíticas freudianas para revelar as motivações ocultas dos consumidores. No final da década de 50 , sua metodologia de pesquisa veio a ser chamada de pesquisa qualitativa.

${ }^{8}$ Ambiente pode ser natural (no ambiente real, ex. Supermercado) ou artificial (elabora-se, em laboratório, um minicinema). A gravação/obtenção dos dados pode ser mecânica (com uso de aparelhos) ou pessoal (pelo próprio pesquisador).

${ }^{9}$ Técnicas para registros dos fenômenos a serem observados. Psicogalvanômetro mensura as respostas do corpo, por meio de eletrodos, a determinados estímulos, Contadores de tráfico medem quanto, em qual lugar e em quais momentos existe maior movimentação pessoal (ex. uso em feiras/congressos), Pupilômetro/oculômetro verifica as reações dos olhos a propagandas e outros estímulos, e Intensidade de voz mensura a magnitude da voz em um dado local (MALHOTRA, 2000).

${ }^{10}$ A técnica ZMET é a primeira técnica de pesquisa de marketing patenteada nos EUA. Foi criada pelo Dr. Gerald Zaltman em conjunto com Robin Higie Coulter. A utilização desse processo ou uso de determinados elementos é proibida, a não ser com uma autorização expressa da Olson Zaltman Associates Company.

${ }^{11}$ ZALTMAN, G.; OLSON, J. Overview of ZMET Research Process. Unpublished Material. By Olson Zaltman Associates, p. 1-13.

${ }^{12}$ Repertory Grid uma construção conceitual dos construtos sem a direta obtenção dos conceitos e de suas estruturas e relações. Começa-se de um ponto para análise e refinamento e continua comparando-os, em alguns casos utiliza-se de atributos opostos (Ex. gostoso/ruim). Laddering emprega uma aplicação sistemática para estruturar associações cognitivas pela análise da cadeia de meio e fins (means-end), seu objetivo é encontrar atributos, conseqüências e valores.

${ }^{13} \mathrm{O}$ termo autodriving muito utilizado nesse tipo de pesquisa indica que o entrevistado é dirigido (driving) pelas informações que estão sendo vistas e ouvidas em seu comportamento (HEISLEY; LEVY, 1991).

\section{ReferênCIAs Bibliográficas}

AAKER, D.A.;

KUMAR, V.;

DAY, G. S.

Pesquisa de Marketing. 1. ed. São Paulo: Atlas, 2001. p.756.

\section{ALLISON, O.}

ACR 2003 Film Festival Review. In: RUSSEL, B.; ROBERT, K. (Org). ACR 2003. Toronto-Canada. Proceedings... Toronto-Canada: North American Conference. Disponível em: <http://www.acrweb.org/acr2003/ ff_review.html>. Acesso em: 22 apr. 2004.

BACELLAR, A.

A Análise de Dados na Pesquisa Qualitativa. Revista Episteme, Tubarão-SC: Editora Unisul, v.7, n.21, p.07-20, jul./out. 2000. 
BALL, M. S.;

SMITH, G. W. H.

Analyzing Visual Data. Qualitative Research Methods Series, 24, Newbury Park, CA: Sage, 1992. p. 88.

CAHILL, D. J.

When to use Qualitative Methods: how about at the midpoint? Marketing News, Chicago, IL, v.32, n.1, p.15-17, jan. 1998.

CATERRALL, $M$.

Academics, practitioners and qualitative market research.

Qualitative Market Research: An International Journal, Bradford, MCB University Press, v.1, n.2, p-69-76, 1998.

CHAUVEL, M.A.

Insatisfação e queixa a empresa: investigando os relatos dos consumidores. In: ENCONTRO ANUAL DA ANPAD, 24., 2000: Florianópolis. Anais... Florianópolis: ANPAD, 2000.

COULTER, R.A.;

ZALTMAN, G.;

COULTER, K. S.

Interpreting Consumer Perceptions of Advertising: An Application of the Zaltman Metaphor Elicitation Technique. Journal of Advertising, Atlanta, v. XXX, n. 4, winter, p.1-21, 2001.

COULTER, R. H.;

ZALTMAN, G.

Using the Zaltman Metaphor Elicitation Technique to Understand Brand Images. In: ALLEN, C.T.; ROEDDER, D. J., (Eds.). Advances in Consumer Research, v.21, Provo, UT: Association for Consumer Research, p.501-507, 1994.
DAY,E.

Quantitative Research Course Emphasizes Understading Merits and Limitations. Marketing News, Chicago, IL,v.16, n.31, p. 9, aug. 1997.

DELBÈS, R.;

GRAMONT, E. T.

Études de Marché, Outil de la

Décision. 1. ed. Paris: Delmas, 1983.

p. 315.

DICHTER, E.

Handbook of Consumer Motivations: philosophy of the worlds of Objects. 1. ed., New York: McGraw-Hill, 1964.

ENGLIS, B. B.;

SOLOMON, M. R.

A Web-Based Methodology for Visually-Oriented Consumer Research. Journal of Interactive Marketing, Direct Marketing Educational Foundation , n.14, winter, p. 1-13, 2000.

FREITAS, H. M. R. et al.

Pelo resgate de alguns princípios da análise de conteúdo: aplicação prática qualitativa em Marketing. In: ENCONTRO ANUAL DA ANPAD, 20., 1996, Angra dos Reis. Anais... Angra dos Reis, ANPAD, 1996.

FREITAS, H. M. R.;

JANISSEK, R.

Análise Léxica e Análise de Conteúdo: técnicas complementares, seqüenciais e recorrentes para exploração de dados qualitativos. Porto Alegre/RS: Sphinx - Sagra (distrib.), julho 2000. p. 176. 
FREITAS, H. M. R. et al.

O Método de Pesquisa Survey. Revista deAdministração-RAUSP, São Paulo, v.35, n.3, p.105-112, jul./ set. 2000.

GABRIEL, C.

The Validity of Qualitative Market Research. Journal of the Market Research Society, London, n.32, p.507-519, oct. 1990.

GAREE, M.L.;

SCHORI, T. R.

Focus Group illuminate Quantitative Research. Marketing News, Chicago, IL, v.31, n.12, p.H25, june 1997.

GONÇALVES, K. P.

Working with the Kano method: Technique helps focus the product development process. Quirk's Marketing Research Review. n. 0588, may 2000. Disponível em: $<$ http:// www.quirks.com>. Acesso em: 13 nov. 2002

GONÇALVES, K. P.;

GONÇALVES, M. P.

Hearing what they say: use of the Kano method keeps Honeywell attuned to the voice of the customer. Quirk's Marketing Research Review.n. 0674, apr., 2001, Disponível em: <http:// www.quirks.com>. Acesso em: 13 nov. 2002.

\section{GOULD, L. C. et al.}

Connections: From the Heroin World. 1. ed. New Haven, CT: Yale University Press, 1974. p. 236.
HARTLEY, R. F.;

PROUGH, G. E.;

FLASCHNER, A. B.

Essentials of Marketing Research.

1. ed. Oklahoma: PennWell Books, 1983. p. 566.

HEISLEY, D. D.;

LEVY, S. J.

Autodriving: a Photoelecitation Technique. Chicago, Journal of Consumer Research, Chicago, IL, n. 18, p.257-272, dec. 1991.

Visual Research: Currente Bias and Future Direction. In: GILLY, M. C.; MEYERS-LEVY, J.(Org.). Advances in Consumer Research, 28, Provo, UT: Association for Consumer Research, p. 45-46, 2001.

\section{HINES, T.}

An evaluation of two qualitative methods (Focus Group Interviews and Cognitive Maps) for Conducting Research Into Entrepreneurial Decision Making. Qualitative Market Research: An International Journal, Bradford, MCB: University Press, v.3, n.1, p.7-16, 2000.

HOLBROOK, M. B.

An Audiovisual Inventory of Some Fanatic Consumer Behavior: the saint tour of a jazz collector's home. In: $\mathrm{M}$. WALLENDORF, P. ANDERSON, (Eds.), Advances in Consumer Research, 14, Provo, UT: Association for Consumer Research, p.144149, 1987. 
Steps Toward a Psychoanalytic Interpretation of Consumption: A Meta Analysis of Some Issues Raised by the Consumer Behavior Odyssey. In: M. J.HOUSTON. (Ed.). Advances in Consumer Research, 15, Provo, UT: Association for Consumer Research, p.537 - 542, 1988.

HUNT, S. D.

Modern Marketing Theory: Critical Issues in the Philosophy of Marketing Science. 1. ed. Cincinnati: South-Western Publishing, 1991. p. 496.

\section{KOTLER, P.}

Administração de Marketing: a edição do novo milênio. 10. ed. São Paulo: Prentice-Hall, 2000. p. 764.

\section{KRAFT, S.;}

NIQUE, W.

Desvendando o Consumidor através das Metáforas: uma aplicação da Zaltman Metaphor Elicitation Technique (ZMET) In: ENCONTRO ANUAL DA ANPAD, 26., 2002, Salvador. Anais...Salvador: ANPAD, 2002.

\section{KURSINA, B.}

Use Vídeos to Obtain Crucial POP info. Marketing News, Chicago, IL, Special Report, n. 20, v.34, p.16-20, nov. 2000.

\section{LEVINSON,A.}

Semantic Attribution as a Means of Understanding the Basics of Local Culture. In: ESOMAR - WORLD ASSOCIATION OF OPINION AND MARKETING RESEARCH PROFESSIONALS., Athens. Proceedings... Athens, 1999.
MALHOTRA, N. K.

Pesquisa de Marketing: uma orientação aplicada. 3. ed. Porto Alegre: Bookman, 2001. p. 720.

\section{MARCHETTI, R. Z.}

Diversidade e Tendências das Pesquisas Qualitativas de Marketing. In: Caderno de Ciências Sociais Aplicada. p.15-24, Curitiba: Editora UFPR, 1995.

\section{MATTAR, F. N.}

Pesquisa de Marketing. Edição Compacta. 5. ed. v.1, São Paulo: Editora Atlas, 1999. p. 276.

McBRIDE, B.

Measuring Print Advertising. American Marketing Association, MarketingPower.com, 2001. Disponível em: <http://www.ama.org>. Acesso em: 13 nov. 2002.

NELEMS, J. H.

Qualitative Research Overview. American Marketing Association, MarketingPower.com, 2001. Disponível em: <http://www.ama.org>. Acesso em: 13 nov. 2002.

NELSON, J. E.;

FRONTEZAK, N.

How Acquaintanceship and Analyst Can Influence Focus Group Results. Journal of Advertising, American Marketing Association, Atlanta, n.17, p.41-48, 1988. 
NICITA, C.;

WALTERS, C.

Hearing the customer's voice: Customer-Driven Concept and Product Development. Quirk’s Marketing Research Review. n. 0555, jan. 2000. Disponívelem: <http://www.quirks.com>. Acesso em: 13 nov. 2002.

NOTESS, G. R.

The Internet as an On-line Service: Bibliographic Databases on the Net. Database, v. 19, n.4, p.92-95, ago./set. 1996.

\section{OLIVEIRA, M.;}

FREITAS, H. M. R.

Focus Group - Pesquisa Qualitativa: resgatando a teoria, instrumentalizando o seu planejamento. Revista de Administração - RAUSP, São Paulo, v.33, n.3, p.83-91, jul./set. 1998.

PERIN, M. G. et al.

A pesquisa survey em artigos de marketing no Enanpad na década de 90. In: ENCONTRO ANUAL DA ANPAD, 24., 2000, Florianópolis. Anais... Florianópolis: ANPAD, 2000

PARKS, A.

On-line Focus Groups Reshape Market Research Industry. Marketing News, Chicago, IL, p. 28, 12 may 1997.

PERRIEN, J.; CHERÓN, E. J.;

ZINS, $\mathrm{M}$.

Recherché en Marketing: Méthodes Décisions. 1. ed. Montréal: Gaëtan Morin, 1984. 615p.

ROLLER, M.

Qualitative Researchers as Methodologists Quirk’s Marketing
Research Review. n. 0744, dec. 2001. Disponível em: <http:// www.quirks.com>. Acesso em: 13 nov. 2002.

ROOK, D. W.;

LEVY, S. J.

Psychosocial Themes in Consumer Grooming Rituals. In: BAGOZZI, R., TYBOUT, A. (Eds.). Advances in Consumer Research, v.10, Provo, UT: Association for Consumer Research, p. 328-333, 1983.

ROSSI, C.A. V.;

SLONGO, L. A.

Pesquisa de Satisfação de Clientes: o estado-da-arte e proposição de um método brasileiro. In: ENCONTRO ANUAL DAANPAD, 21., 1997, Rio de Janeiro. Anais... Rio de Janeiro: ANPAD, 1997.

\section{SHETH, N. J.;}

MITTAL, B.;

NEWMAN, I. B.

Comportamento do Cliente: Indo além do Comportamento do Consumidor. 1. ed. São Paulo: Atlas, 2001. p.796.

SCHIFFMAN, L. G.;

KANUK, L. L.

Comportamento do Consumidor, 6. ed. Rio de Janeiro: Editora LTC, 2000. p. 476.

SOARES, V. M. S.;

COSENZA, O. N.;

GOMES, C. F. S.

Técnicas Qualitativas e “soft Systems Methodology” Aliadas ao Enfoque Sistêmico. Revista de Administração-RAUSP, São Paulo, v.36, n. 3, p.100-107, jul./set., 2001. 
ZALTMAN, G.

Metaphorically Speaking: New Technique uses Multidisciplinary ideas to Improve Qualitative Research. Marketing Research, Chicago, IL, v. 8, n. 2, p. 13-20, summer 1996.

Rethinking Market Research: Putting People Back in. Journal of Marketing Research, Chicago, IL, XXXIV, p. 424-437, nov. 1997.

Consumer Researchers: Take a Hike! Journal of Consumer Research, Chicago, IL, v, 26, p. 423-428, mar. 2000.
ZALTMAN, G;

COULTER, R. H.

Seeing the Voice of the Customer: Metaphor-Based Advertising

Research. Journal of Advertising Research, Advertising Research Foundation, New York, v. 35, n.4, p.3551, 1995.

ZIMMERMAN, A. S.;

SZENBERG, $M$.

Implementing International Qualitative Research: Techniques and Obstacles. Qualitative Market Research: An International Journal, Bradford, MCB University Press, v.3, n.3, p.158164, 2000. 